\title{
A STABLE GROUP-PAIR INVARIANT OF THREE-DIMENSIONAL MANIFOLDS
}

\author{
DALE ROLFSEN
}

\begin{abstract}
A new algebraic invariant of 3-manifolds is discussed. It is an application of the rational surgery calculus.
\end{abstract}

Every closed oriented 3-manifold $M$ may be obtained from the 3-sphere $S^{3}$ by Dehn's surgery construction $\left[\mathbf{D}, \mathbf{L}, \mathbf{W}_{\mathbf{1}}\right]$. We describe how the surgery data for this construction determine a group pair $(\bar{G}, \bar{H})$. Our main result is that if two different surgery data actually describe the same 3-manifold (up to orientation-preserving homeomorphism), then their associated group pairs are stably isomorphic in a sense defined below. Thus the stable equivalence class of $(\bar{G}, \bar{H})$ is an algebraic invariant of $M$ itself. Since this class determines $\pi_{1}(M)$, it is a priori at least as sensitive an invariant as the fundamental group.

S. Fukuhara $[\mathbf{F}]$ made a similar construction for integrally framed links and proved a similar theorem, based on Kirby's calculus of links [K]. Our result was motivated by, and generalizes, Fukuhara's theorem, and provides a "coordinate-free" proof. It is based on the rational link calculus $\left[\mathbf{R}_{\mathbf{1}}, \mathbf{R}_{\mathbf{2}}\right]$ for 3 -manifolds. I would like to thank Professor K. Murasugi for bringing Fukuhara's paper to my attention.

Surgery data. We work in the PL category (although the results are equally valid in the smooth and tame topological categories) and endow $S^{3}$ with a fixed orientation. Surgery data in $S^{3}$ consist of a collection $V=V_{1} \cup \cdots \cup V_{n}$ of disjoint solid tori $V_{i} \cong S^{1} \times D^{2}$ embedded in $S^{3}$, together with Jordan curves $J=J_{1} \cup \cdots \cup J_{n}$ such that $J_{i}$ is an essential (= nonseparating) curve in the boundary torus of $V_{i}$. The surgery manifold $M^{3}=M(V, J)$ is obtained by deleting the interiors of the $V_{i}$ and replacing each of them by a solid torus in such a way that $J_{i}$ is attached to a meridian $\left(* \times S^{1}\right.$ in $\left.S^{1} \times D^{2}\right)$. As is well known, $M$ is uniquely determined by $(V, J)$ and inherits an orientation.

The surgery group pair. Given surgery data $(V, J)$ in $S^{3}$, consider the group $G=\pi_{1}\left(S^{3}-V\right)$, which is just the link group associated with the link of central curves of $V$. The curves $J_{i}$ represent elements of $G$, defined up to conjugacy, so we may consider the subgroup $H=\left\{J_{1}, \ldots, J_{n}\right\}_{G}$, that is, the normal closure of the classes of the $J_{i}$ in $G$. Now the subgroup $[G, H]$, generated by commutators $[g, h]=g h g^{-1} h^{-1}, g \in G, h \in H$ is normal in $G$, so we consider the quotient group $\bar{G}=G /[G, H]$. Also $[G, H]$ is a subgroup of $H$ so we let $\bar{H}=H /[G, H]$.

Received by the editors August 28, 1982 and, in revised form, April 18, 1983.

1980 Mathematics Subject Classification. Primary 57N10; Secondary 57M05, 20F12. 
We shall call this pair of groups $(\bar{G}, \bar{H})$ the surgery group pair associated with the data $(V, J)$. Note that $\bar{H}$ is abelian and is central in $\bar{G}$. One may verify that $\pi_{1}(M) \cong G / H \cong \bar{G} / \bar{H}$, so the surgery group pair determines the fundamental group of $M=M(V, J)$.

Statement of results. Let $A, B$ and $C$ be groups with $B$ a subgroup of $A$. Then $(A, B) \times C$ denotes the group pair $(A \times C, B \times C)$, where $B \times C$ is the obvious subgroup of the direct product $A \times C$. We say that two group pairs $\left(A_{1}, B_{1}\right)$ and $\left(A_{2}, B_{2}\right)$ are stably isomorphic if there are integers $r, s \geqslant 0$ and an isomorphism of pairs

$$
\left(A_{1}, B_{1}\right) \times \mathbf{Z}^{r} \cong\left(A_{2}, B_{2}\right) \times \mathbf{Z}^{s},
$$

where $\mathbf{Z}^{r}$ denotes the free abelian group of rank $r$. We may now state our main result.

Let $(V, J)$ and $\left(V^{\prime}, J^{\prime}\right)$ denote surgery data in $S^{3}$ and let $(\bar{G}, \bar{H})$ and $\left(\bar{G}^{\prime}, \bar{H}^{\prime}\right)$ be their respective surgery group pairs.

THEOREM 1. If the manifolds $M(V, J)$ and $M\left(V^{\prime}, J^{\prime}\right)$ are orientation-preserving homeomorphic, then $(\bar{G}, \bar{H})$ and $\left(\bar{G}^{\prime}, \bar{H}^{\prime}\right)$ are stably isomorphic.

We note that even the stable isomorphism class of $(\bar{G}, \bar{H})$ determines $\pi_{1}(M)$. There is a partial converse to the theorem in the case of irreducible sufficiently large 3-manifolds. For Waldhausen $\left[\mathbf{W}_{2}\right]$ has shown that for this class of manifolds, the fundamental group determines the (unoriented) homeomorphism type.

Proof of Theorem 1. The proof is an application of the main theorem of $\left[\mathbf{R}_{\mathbf{2}}\right]$. For the reader's convenience, we outline the pertinent results. Consider the surgery data $(V, J), V=V_{1} \cup \cdots \cup V_{n}, J=J_{1} \cup \cdots \cup J_{n}$. There are three "moves" in the rational surgery calculus by which these data give rise to another set $\left(V^{\prime}, J^{\prime}\right)$ of surgery data.

Move 1. Let $h: S^{3} \rightarrow S^{3}$ be an orientation-preserving homeomorphism and define $V^{\prime}=h(V), J^{\prime}=h(J)$.

Move 2. Locate (if possible) a solid torus, say $V_{i}$, of $V$ whose closed complement in $S^{3}$ is also a solid torus $W=S^{3}-V_{i}$. Let $g: W \rightarrow W$ be a "twist" homeomorphism (corresponding to $(x, y) \mapsto(x, x y)$ in complex notation for $\left.S^{1} \times D^{2} \subset C \times C\right)$. Define $V_{i}^{\prime}=V_{i}, V_{j}^{\prime}=g\left(V_{j}\right)$ for $j \neq i$, and $J^{\prime}=g(J)$.

Move 3. Let $V_{0}$ be a solid torus disjoint from $V$, and let $J_{0}$ be a meridian of $V_{0}$. Define $V^{\prime}=V_{0} \cup V$ and $J^{\prime}=J_{0} \cup J$.

Let $\leftrightarrow$ denote the equivalence relation among surgery data generated by moves $1-3$ and their inverse moves. The principal result of $\left[\mathbf{R}_{2}\right]$ is

THEOREM 2. The manifolds $M(V, J)$ and $M\left(V^{\prime}, J^{\prime}\right)$ are orientation-preserving homeomorphic if and only if $(V, J) \leftrightarrow\left(V^{\prime}, J^{\prime}\right)$.

This reduces the proof of Theorem 1 to checking the effect of moves $1-3$ on the surgery group pair $(G, H)$ determined by $(V, J)$. It is easily seen that if $\left(V^{\prime}, J^{\prime}\right)$ arises from $(V, J)$ by Move 1 or 2 , then the surgery group pair $\left(\bar{G}^{\prime}, \bar{H}^{\prime}\right)$ is actually isomorphic with $(\bar{G}, \bar{H})$. The proof is completed by the following lemma. 
Lemma. Suppose $\left(V^{\prime}, J^{\prime}\right)$ and $(V, J)$ are as in the description of Move 3, and let $\left(\bar{G}^{\prime}, \bar{H}^{\prime}\right)$ and $(\bar{G}, \bar{H})$ denote their respective surgery group pairs. Then there is an isomorphism $\left(\bar{G}^{\prime}, \bar{H}^{\prime}\right) \cong(\bar{G}, \bar{H}) \times \mathbf{Z}$.

Proof of the Lemma. We will show, moreover, that the inclusion homomorphism $i_{*}: G^{\prime}=\pi_{1}\left(S^{3}-V^{\prime}\right) \rightarrow G=\pi_{1}\left(S^{3}-V\right)$ induces the natural projection $\bar{G}^{\prime} \cong \bar{G} \times$ $\mathbf{Z} \rightarrow \bar{G}$ in the direct product, under the isomorphism. Consider the exact sequence of groups

$$
1 \rightarrow K \rightarrow G^{\prime} \stackrel{i}{\rightarrow} G \rightarrow 1
$$

By standard geometric arguments, $i_{*}$ is indeed surjective and its kernel $K$ is the normal closure of the class of $J_{0}$ in $G^{\prime}$, since it is a meridian of $V_{0}$. Thus we have $K=\left\{J_{0}\right\}_{G^{\prime}}$ and, by definition, $H^{\prime}=\left\{J_{0}, J_{1}, \ldots, J_{n}\right\}_{G^{\prime}}, H=\left\{J_{1}, \ldots, J_{n}\right\}_{G}$. Clearly $i_{*}\left(H^{\prime}\right)=H$ and so $i_{*}$ induces a (surjective) homomorphism

$$
\bar{G}^{\prime}=G^{\prime} /\left[G^{\prime}, H^{\prime}\right]^{\bar{i}} \stackrel{\text { * }}{\rightarrow} \bar{G}=G /[G, H] .
$$

Letting $\bar{K}=\operatorname{ker} \bar{i}_{*}$ we have the exact sequence

$$
1 \rightarrow \bar{K} \rightarrow \bar{G}^{\prime} \rightarrow \bar{G} \rightarrow 1 .
$$

It is easily seen that $\bar{K}$ is just the projection of $K$, and since the class $\bar{J}_{0}$ of $J_{0}$ in $\bar{G}^{\prime}$ is central, we see that $\bar{K}$ is a cyclic group, generated by $\bar{J}_{0}$. Moreover, $\bar{J}_{0}$ cannot have finite order, since under abelianization of $G^{\prime}$ (hence of $\bar{G}^{\prime}$ ) it has infinite order (corresponding to a nontrivial element in $\left.H_{1}\left(S^{3}-V^{\prime}\right) \cong \mathbf{Z}^{n+1}\right)$. Therefore $\bar{K} \cong \mathbf{Z}$, generated by $\bar{J}_{0} \in \bar{G}^{\prime}$. Moreover, $\bar{K}$ is central in $\bar{G}^{\prime}$.

Next we argue that the sequence $(0)$ splits. That is, there is a homomorphism $f$ : $\bar{G} \rightarrow \bar{G}^{\prime}$ such that $\bar{i}_{*} \circ f=$ identity. We describe $f$ geometrically. Let $g$ be a loop in $S^{3}-V$ representing (by abuse of notation) $g \in G$ and $\bar{g} \in \bar{G}$. By a general position argument and adjunction of an appropriate multiple of the meridian $J_{0}$, one can find a loop $g^{\prime}$ in $S^{3}-V^{\prime}$ satisfying

$$
g^{\prime} \text { is homotopic with } g \text { in } S^{3}-V
$$

and

$$
g^{\prime} \text { is homologically trivial in } S^{3}-V_{0} \text {. }
$$

Define $f(\bar{g})=\bar{g}^{\prime}$, where $\bar{g}^{\prime}$ is the image of $g^{\prime}$ under the projection $G^{\prime} \rightarrow \bar{G}^{\prime}$. Once we have checked that $f$ is well defined, it is easy to see that $f$ is a homomorphism, and $i_{*} \circ f=$ identity follows from (1).

We need to show that if a loop $g_{1}$ in $S^{3}-V$ also represents $\bar{g} \in \bar{G}$ and a loop $g_{1}^{\prime}$ in $S^{3}-V^{\prime}$ satisfies, with $g_{1}$, the conditions (1) and (2), then $\overline{g_{1}^{\prime}}=\overline{g^{\prime}}$. Since $g_{1} g^{-1}$ lies in $[G, H]$, and $i_{*}\left[G^{\prime}, H^{\prime}\right]=[G, H]$, we have $g_{1} g^{-1}=i_{*}(c)$ for some $c \in\left[G^{\prime}, H^{\prime}\right]$. Also, since $i_{*}\left(g_{1}^{\prime}\right)=g_{1}$, and $i_{*}\left(g^{\prime}\right)=g$ we have $i_{*}\left(g_{1}^{\prime}\left(g^{\prime}\right)^{-1}\right)=i_{*}(c)$ and hence

$$
g_{1}^{\prime}\left(g^{\prime}\right)^{-1}=c k, \quad \text { where } c \in\left[G^{\prime}, H^{\prime}\right] \text { and } k \in K \text {. }
$$


But $K$ is normally generated by $J_{0}$, considered in $G^{\prime}$, so we have an equation

$$
k=\prod_{i=1}^{r} a_{i} J_{0}^{\varepsilon_{1}} a_{i}^{-1}, \quad \text { where } a_{i} \in G^{\prime} .
$$

Since $g_{1}^{\prime}\left(g^{\prime}\right)^{-1}$ is also homologically trivial in $S^{3}-V_{0}$, as is the product of the commutators $c$, we conclude from (3) and (4) that the exponent sum must be zero: $\sum_{i=1}^{r} \varepsilon_{i}=0$. Now projecting into $\bar{G}^{\prime}$, where $\bar{J}_{0}$ is central, equation (3) implies that in

$$
\overline{g_{1}^{\prime}}{\overline{\left(g^{\prime}\right)}}^{-1}=\bar{c} \bar{k}=\bar{k}=J_{0}^{\sum \varepsilon_{i}}=1,
$$

and we have established that $f$ is well defined.

Now we know the sequence $(0)$ is split and $\bar{K}$ is central, so the map $\alpha$ : $\bar{G} \times \bar{K} \rightarrow \bar{G}^{\prime}$ given by $\alpha(x, y)=f(x) y$ is easily seen to be an isomorphism. We leave the reader to verify that $\alpha(\bar{H} \times \bar{K})=\bar{H}^{\prime}$. This, together with the isomorphism $\bar{K} \cong \mathbf{Z}$, proves the lemma. The proof of Theorem 1 is now complete.

Examples and remarks. It is natural to ask under what conditions stable isomorphism of groups implies actual isomorphism. I am indebted to Professor Al Hales for the following simple example of groups $G_{1}, G_{2}$ which are nonisomorphic, yet $G_{1} \times \mathbf{Z} \cong G_{2} \times \mathbf{Z}$

EXAMPLE 1. Let $G_{1}=\left\langle x, y \mid x^{11}=1, y x y^{-1}=x^{2}\right\rangle$ and $G_{2}=\langle x, y| x^{11}=$ $\left.1, y x y^{-1}=x^{8}\right\rangle$, so each is an extension of a cyclic group of order 11 by an infinite cyclic group, with only the action of the latter on the former differing. The reader will enjoy checking that $G_{1}$ and $G_{2}$ have the required properties.

In the next two examples we adopt the notation of the rational link calculus (see $\left[\mathbf{R}_{1}\right.$ or $\left.\mathbf{R}_{2}\right]$ ) to depict surgery data in $S^{3}$. The link drawn denotes the central curves of the surgery solid tori and the coefficient $p / q$ means that the curve on the boundary torus which equals $p$ meridians plus $q$ longitudes is to be killed in the surgery ( $p, q$ coprime).

EXAMPLE 2. The lens space $L(p, q)$ has the two surgery descriptions:

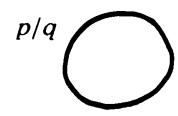

Description I

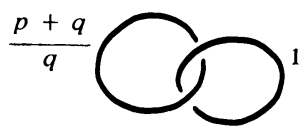

Description II

With Description I we have $G_{\mathrm{I}} \cong \bar{G}_{\mathrm{I}} \cong \mathbf{Z}$ and $H_{\mathrm{I}} \cong \bar{H}_{\mathrm{I}} \cong p \mathbf{Z} \subset \mathbf{Z}$. With Description II, $G_{\mathrm{II}} \cong \bar{G}_{\mathrm{II}} \cong \mathbf{Z} \times \mathbf{Z}$, and $H_{\mathrm{II}} \cong \bar{H}_{\mathrm{II}}$ is the subgroup of $\mathbf{Z} \times \mathbf{Z}$ spanned by $(p+q, q)$ and $(1,1)$. One can verify easily in this case that $\left(\bar{G}_{\mathrm{II}}, \bar{H}_{\mathrm{II}}\right) \cong\left(\bar{G}_{\mathrm{I}}, \bar{H}_{\mathrm{I}}\right) \times$ $\mathbf{Z}$.

It seems difficult to determine in general when two groups (or group pairs) are not stably isomorphic. The following provides one test. Its proof is simple.

Proposition. If $G_{1}$ and $G_{2}$ are stably isomorphic (that is, $G_{1} \times \mathbf{Z}^{r} \cong G_{2} \times \mathbf{Z}^{s}$ ), then $G_{1} / Z\left(G_{1}\right)$ is isomorphic with $G_{2} / Z\left(G_{2}\right)$, where $Z(G)$ denotes the center of the group $G$. 
We can apply this to see that the group $\bar{G}$ alone is sufficient to distinguish the various Brieskorn manifolds $\Sigma(p, q, r)$.

EXAmple 3. Let $1<p<q<r$ be coprime integers. Define the following subset of complex 3-space:

$$
\Sigma(p, q, r)=\left\{\left(Z_{1}, Z_{2}, Z_{3}\right) \mid Z_{1}^{p}+Z_{2}^{q}+Z_{3}^{r}=0 \text { and } \sum\left|Z_{i}\right|^{2}=1\right\} .
$$

This orientable 3-manifold may be constructed from the following surgery data where $l, m, n$ are any integers solving the equation $l q r+m p r+n p q=1$ (see $[\mathbf{C}-\mathbf{H}])$.

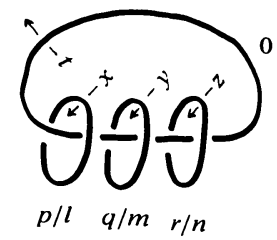

We compute the link group, in terms of the generators pictured.

$$
\begin{gathered}
G=\langle x, y, z, t| t \text { commutes with } x, y, z\rangle, \\
H=\left\{x^{p} t^{l}, y^{q} t^{m}, z^{r} t^{n}, x y z\right\}_{G} .
\end{gathered}
$$

Therefore

$$
\left.\bar{G}=\langle x, y, z, t| t, x^{p} t^{l}, y^{q} t^{m}, z^{r} t^{n}, x y z \text { are all central }\right\rangle .
$$

On killing these central elements we obtain the well-known triangle group which has no center, so

$$
\bar{G} / Z(\bar{G}) \cong\left\langle x, y, z \mid x^{p}=y^{q}=z^{r}=x y z=1\right\rangle .
$$

Since these are known to be nonisomorphic for different values of $p, q$, $r$, we verify that the various Brieskorn manifolds are distinguished by the stable class of their group $\bar{G}$.

The discerning reader will have noticed that $\pi_{1}$ would have served equally well in the above example. We close with some questions.

Question 1. Are there two 3-manifolds with isomorphic fundamental groups, yet which can be distinguished by their surgery group pairs? By Example 2, lens spaces do not qualify (see addendum).

Question 2. Can one ever distinguish $M$ from $-M$ (reversed orientation) using the surgery group pair, together with other surgery-related data?

Question 3. Are there other algebraic invariants which may be derived from the surgery group pair and are more easily computed and compared? What about lower central series and other commutator-defined subgroups, which are unchanged under stable isomorphism?

ADDENDUM. I would like to thank the referee for pointing out that the answer to Question 1 is "no". Here is his argument, which he attributes to Cameron Gordon. A similar argument was shown to me by R. Litherland.

Let $\Pi=\pi_{1}(M)$, and fix a finite presentation

$$
1 \rightarrow R \rightarrow F \rightarrow \Pi \rightarrow 1 .
$$


Let $\bar{F}=F /[F, R]$ and $\bar{R}=R /[F, R]$. There is a commutative diagram with exact rows.

$$
\begin{array}{lllllllll}
1 & \rightarrow & R & \rightarrow & F & \rightarrow & \Pi & \rightarrow & \\
& & \downarrow & & \downarrow & & \| & & \\
1 & \rightarrow & H & \rightarrow & G & \rightarrow & \Pi & \rightarrow & 1
\end{array}
$$

Passing to the 5-term exact homology sequences gives:

$$
\begin{array}{ccccccccc}
0=H_{2}(F) & \rightarrow & H_{2}(\Pi) & \rightarrow & \bar{R} & \rightarrow & H_{1}(F) & \rightarrow & H_{1}(\Pi) \rightarrow 0 \\
\downarrow & & \| & & \downarrow & & \downarrow & & \| \\
H_{2}(G) & \stackrel{0}{*} & H_{2}(\Pi) & \rightarrow & \bar{H} & \rightarrow & H_{1}(G) & \rightarrow & H_{1}(\Pi) \rightarrow 0
\end{array}
$$

$\left(H_{2}(G) \rightarrow H_{2}(\Pi)\right.$ is 0 because $H_{2}(G)=H_{2}\left(S^{3}-V\right) / \pi_{2}\left(S^{3}-V\right)$.) It follows that there is an isomorphism $g: \bar{R} \oplus H_{1}(G) \rightarrow \bar{H} \oplus H_{1}(F)$. It is easy to see that the diagram

$$
\begin{aligned}
& 0 \quad \rightarrow \quad \bar{R} \oplus H_{1}(G) \quad \rightarrow \quad \bar{F} \oplus H_{1}(G) \quad \rightarrow \quad \Pi \quad \rightarrow \quad 1 \\
& g \downarrow \quad f \downarrow \quad \| \\
& 0 \quad \rightarrow \quad \bar{H} \oplus H_{1}(F) \quad \rightarrow \quad \bar{G} \oplus H_{1}(F) \quad \rightarrow \quad \Pi \quad \rightarrow \quad 1
\end{aligned}
$$

can be filled in; $f$ is an isomorphism by the 5-lemma. Hence $(\bar{G}, \bar{H})$ is stably isomorphic to $(\bar{F}, \bar{R})$, and so depends (up to stable isomorphism) only on $\Pi$.

\section{REFERENCES}

[C-H] A. Casson and J. Harer, Some homology lens spaces which bound rational homology balls, Pacific J. Math. 96 (1981), 23-36.

[D] M. Dehn, Uber die Topologie des drei-dimensionalen Raumes, Math. Ann. 75 (1910), 402-413.

[F] S. Fukuhara, On framed link groups, preprint.

[K] R. Kirby, A calculus for framed links in $S^{3}$. Invent. Math. 45 (1978), 35-56.

[L] W. B. R. Lickorish, A representation of orientable combinatorial 3-manifolds, Ann. of Math. (2) 76 (1962), 531-538.

[R $\left.\mathbf{R}_{1}\right]$ D. Rolfsen, Knots and links, Publish or Perish, 901 Washington St., Wilmington, Deleware 19801. 1976.

$\left[\mathbf{R}_{\mathbf{2}}\right]$ _. Rational link calculus: extension of Kirby's theorem, Pacific J. Math. (to appear).

[W $]$ A. Wallace, Modifications and cobounding manifolds, Canad. J. Math. 12 (1960), 503-528.

$\left[\mathbf{W}_{2}\right]$ F. Waldhausen, On irreducible 3-manifolds which are sufficiently large, Ann. of Math. (2) 87 (1968), 56-88.

Department of Mathematics, The University of British Columbia, Vancouver, British ColUMBIA, CANADA V6T 1Y4 